\title{
Ia zona desnuclearizada latinoamericana en la perspectiva de la cooperación regional
}

\section{INTRODUCCIÓN}

E1 Tratado de Tlatelolco abierto a la firma el 14 de febrero de 1967, en virtud. del cual se constituyó en América Latina una zona libre de armas nucleares, fue un notable éxito diplomático y polftico. La iniciativa, impulsada principalmente por México y respaldada por los gobiernos de Brasil, Chile, Bolivia y Ecuador, se materializó en la Declaración Conjunta sobre Desnuclearización de América Latina suscrita por los Presidentes de estos países latinoamericanos, el 29 de abril de 1963, en la cual expresaban su deseo de instituir un acuerdo multilateral mediante el cual se comprometerían a establecer una zona desnuclearizada en la región latinoamericana ${ }^{1}$.

El concepto de la Zona Libre de Armas Nucleares emana del interés de los países no nucleares de desprenderse, en función de sus propios objetivos de seguridad, de la espiral armamentista en que se encuentran envueltas las potencias nucleares. Con ese fin un conjunto de Estados que coexisten en un espacio geográfico dado, negocian y suscriben un tratado, que constituye un estatuto de total ausencia de armas nucleares, cuyo ámbito y extensión es específica, y que establece, también, un sistema de verificación y control que garantiza efectivamente su cumplinaiento².

\footnotetext{
'Sobre la historia de la elaboración del Tratado de TlateloIco: Alfonso García Robles, "Mesures de désarmement dans des zones particulières; Le Traité visant l'interdiction des armes nucléaires en Amérique Latine", Académie de Droit International, Recueil des Cours 1971, 1; La desnuclearización de América Latina, El Colegio de México, 1965; The Denuclearization of Latin America, Carnegie Endowment Ior International Peace, 1967; La Proscripción de las Armas Nucleares en la América Latina, EI Colegio Nacional, 1975; "El Tratado. para la proscripción de las armas nucleares en la América Latina", Anuario Mexicano de Relacionés Internacionales, 1980, UNAM, México, 1981.

Héctor Gros Espiell, "Desarme nuclear, perspectivas regionales", en: Revista de Estudios Internacionales, Volumen 2, No 4, octubre-diciembre 1981, Centro de Estudios Constitucionales, Madrid, p. 982.
} 
A pesar de su antigüedad, la zona libre de armas nucleares latinoamericana ha sido por muchos años una realidad excepcional, sólo recientemente se ha conformado una zona desnuclearizada en $\mathrm{el}$ Pacífico Sur en virtud del Tratado de Rarotonga aprobado y abierto a la firma el 6 de agosto de $1985^{3}$.

E1 Tratado de Tlatelolco fue una iniciativa política destinada a escapar al enfrentamiento entre las grandes potencias nucleares en territorio latinoamericano, posibilidad que se había palpado con excesivo realismo en el conflicto de los Misiles en 1962, en el que Estados Unidos y la Unión Soviética se enfrentaron directamente en un escenario regional y existió un peligro real de conflagración nuclear.

Los creadores de Tlatelolco siempre pensaron que esta iniciativa sólo tendría un real efecto si se insertaba como la pieza de un gran rompecabezas que culminaría con el desarme general y completo ${ }^{4}$. No obstante, dos décadas de Naciones Unidas dedicadas al desarme no han resultado ni remotamente exitosas; la carrera armamentista ha recrudecido y los acuerdos y resoluciones condenatorias provenientes de los órganos especializados y de la prcpia Asamblea General no pasan de ser meras declaraciones retóricas. El valor de la zona desnuclearizada debe encontrarse entonces en otro contexto: la constitución de un sistema regional de seguridad y cooperación militar en el que la inexistencia de armas nucleares establece un limite pactado a la carrera armamentista y contribuye a fomentar la confianza entre los Estados que en ella participan. En sus relaciones de seguridad con las potencias extrarregionales la desnuclearización establece un espacio de protección excepcional, toda vez que las potencias nucleares se han comprometido formalmente a no emplazar armas atómicas en territorio latinoamericano, como asimismo, a no usar ni amenazar con el uso de estas armas a los países que la integran.

El tema de la desnuclearización y de la energía nuclear, constituye un elemento muy significativo para la transformación de las relaciones militares regionales desde un esquema de competencia hacia un sistema de cooperación, de allí que, un análisis detallado de sus perspectivas de desarrollo, contribuye a ilustrar los problemas y posibilidades que estos fenómenos introducen en el tema de la se-

-EI Tratado de Rarotonga negociado por las partes fue aprobado el 6 de agosto de 1985 y se encuentra abierto a la firma. "South Pacific Nuclear Free Zone Treaty", en: Law of the Sea Bulletin, N9 6, October, 1985, Office of the Special Representative of the Secretary General of the Law of the Sea, p. 24 y ss. Sobre sus objetivos ver David Lange, "New Zeland's Security Policy", en: Foreign Affairs, Summer 1985, p. 1012.

"Héctor Gros Espiell, "La Seguridad Colectiva en América Latina y el Tratado de Tlatelolco", en: Anuario de Derecho Intemacional, Facultad de Derecho, Universidad de Navarra, Vol. Iv, 1977-1978, p. 18. 
guridad regional con la perspectiva que conceden los 20 afros de vigencia del Tratado de Tlatelolco.

\section{EVALUAGIÓN DE LA ZONA DESNUGLEARIZADA LATINOAMERRTCANA}

Hasta la fecha no existe un consenso respecto a cuáles pueden considerarse los requisitos de constitución de una zona desnuclearizada, sin embargo la aproximación más compartida se encuentra en la definición contenida en el Informe del Grupo de Expertos de las Naciones Unidas comisionado para realizar un estudio amplio sobre las Zonas Libres de Armas Nucleares, de 19755. Dado que la diversidad geográfica y política de las potenciales NWFZ hace imposible una caracterización excesivamente rígida, el Grupo de Expertos acordó señalar los principales requisitos que debe reunir una zona de este tipo para asegurar el objetivo fundamental que consiste en eliminar completamente las armas nucleares dentro del espacio geográfico que la constituye ${ }^{\natural}$. Hecha esta precisión previa, varios principios fueron enunciados:

1) La iniciativa debe gestarse en la propia región que se desnucleariza;

2) Los principios que la sustentan deben negociarse directamente por los Estados que la establecen y deben traducirse en un tratado multilateral que consagra la $N W F Z$ a perpetuidad;

3) Aun cuando la adhesión al Tratado es voluntaria, la NWFZ debe comprender a todos los Estados militarmente significativos de la región;

4) Los tratados previamente existentes en la Zona no deben ser vulnerados por el Tratado que la constituye;

5) Debe establecerse un sistema de verificación y control confiable;

6) Los usos pacíficos de la energía nuclear deben estar permitidos;

7) La Zona debe tener un ámbito geográfico claramente delimitado;

8) En la definición del ámbito territorial las partes deben respetar el derecho internacional, incluyendo la libertad de los mares, los estrechos que se usan para la navegación internacional y el espacio aéreo;

9) La NWFZ debe tener el respaldo de las potencias nucleares.

${ }^{5}$ Alfonso Garcia Robles, La Desnuclearización de América Latina. México, 1965.

${ }^{\circ}$ Comprehensive Study of the Question of Nuclear Weapon-Free Zone in All its Aspects: Special Report of the Conference of the Committee on Disarmament. New York, United Nations, General Assembly Official Records, 1975. 30th Session, Supplement No $27-\mathrm{A}$. 
Teniendo en cuenta estos requisitos que hemos señalado intentaremos realizar una revisión de la NWFZ latinoamericana a fin de comprobar si ella reúne los requisitos que el Grupo de Expertos ha señalado como indispensables para constituir plenamente un estatuto de estas características ${ }^{7}$.

\section{Iniciativa regional}

En 1962 los gobiernos de Brasil, Chile, Bolivia y Ecuador presentaron a las Naciones Unidas un proyecto de resolución contemplando Ia posibilidad de establecer en América Laeina una zona libre de armas nucleares. Posteriormente en 1963 los Presidentes de estos mismos países junto al Mandatario mexicano, suscribieron una declaración conjunta en la cual expresaban su deseo de establecer un acuerdo multilateral que consagrase la desnuclearización regionals. La iniciativa fue llevada a las Naciones Unidas y recibió un decidido respaldo en el organismo internacional. Tomando como base un proyecto presentado por Bolivia, Brasil, Chile, Ecuador, México, Costa Rica, El Salvador, Haití, Honduras, Panamá y Uruguay, se aprobó la Resolución 1911 (XvII) el 27 de noviembre de 1963. En esta Resolución la Asamblea General daba la bienvenida a la iniciativa planteada por los cinco Presidentes respecto a la desnuclearización de América Latina, expresaba la esperanza de que los Estados de la región iniciaran "estudios tendientes a establecer las medidas que deberían acordarse para obtener esa finalidad" y solicitaba a! Secretario General que proporcionara a los países latinoamericanos Ia asistencia que pudieran requerir para establecer definitivamente el estatuto de desnuclearización.

A partir de ese momento, la Cancillería mexicana comenzó a ejercer un papel preponderante en la continuación del proceso, realizando para ello numerosas consultas diplomáticas, destinadas a conocer las posiciones de los distintos países con miras a convocar una conferencia de negociación. La Reunión Preliminar sobre Desnuclearización de América Latina (REUPRAL) se reunió en México entre el 23 y 27 de noviembre de 1964. En esta ocasión se acordó constituix un órgano ad-hoc destinado a elaborar un proyecto de tratado multilateral para la desnuclearización de América Latina.

Atendidos estos antecedentes, puede afirmarse que la iniciativa de constituir una NWFZ en la región fue gestada y propuesta por los

TRamesh Thakur, "A Nuclear-Weapon-Free South Pacific: a New Zealand Perspective", en: Pacific Affairs, Vol. 58, No 2, Summer I985, p. 221 y ss. En este estudio el autor analiza la factibilidad de la iniciativa del Pacífico Sur en relación a los requisitos señalados por el Grupo de Expertos de las Naciones Unidas, metodologia que hemos adoptado para este trabajo.

${ }^{8}$ Alfonso Garcfa Robles, The Latin American Nuclear Free Zone. The Stanley Foundation, Occasional Paper, 19. Mayo, 1979. 
Pilar Armanet Armanet / La zona desnuclearizada latinoamericana en la ...

propios palses latinoamericanos que impulsaron internamente su proceso de formulación y buscaron su aceptación externa presentatido su proposición ante las Naciones Unidas.

\section{Establecimiento de la Zona Desnuclearizada en un Tratado multilateral}

El órgano ad-hoc constituido por la REUPRAt para la formulación de los principios y la elaboración del Tratado denominado Comisión Preparatoria para la Desnuclearización de América Latina (COPREDAL) se reunió por primera vez en México entre el 15 y 22 de marzo de 1965 y luego de cuatro sesiones culminó sus trabajos en febrero de 1967.

El Tratado propuesto recogía los principales objetivos que debian estar presentes en la constitución de una NwFZ latinoamericana, y concluido el proceso de negociación se abrió a la firma de los Estados latinoamericanos el 16 de febrero de 1967.

El Tratado para la Proscripción de las Armas Nucleares en América Latina, más comúnmente conocido como Tratado de Tlatelolco, daba cumplimiento formal al requisito de establecer la zona desnuclearizada mediante un acuerdo jurídico multilateral gestado a partir de una iniciativa propiamente latinoamericana.

La adhesión de todos los paises militarmente relevantes de la región

Aun cuando, como señalábamos anteriormente, la adhesión a un Tratado sólo puede ser voluntaria, existe un gran consenso respecto a la necesidad de que en el acuerdo participen los Estados militarmente relevantes de la región a fin de que la NwFz tenga una base de sustentación política convincente.

Es por ello que en su gestación resultó fundamental contar con el apoyo de Brasil y México, y la participación activa de Argentina en la negociación del Tratado; sin embargo ha sido precisamente en este aspecto donde más dificultades se han encontrado para concretar la desnuclearización regional (Ver Cuadro $N^{\circ} 1$ ).

Desde el inicio de las conversaciones en COPREDat, el problema más agudo fue, sin dudas, el de la puesta en vigencia del Tratado, en torno al cual se plantearon dos posiciones divergentes y que parecían irreconciliables. Para algunos Estados, el Tratado debía entrar en vigencia (para aquellos Estados que lo ratificaran) desde la fecha en que depositaban sus acuerdos de ratificación, de acuerdo con las prácticas usuales en el Derecho Internacional. Para esta pos"ción, el tratado y el Organismo que éste conformara debía entrar en operación desde el momento en que se depositaran once instrumentos de ratificación, número que constituía la mayorfa del grupo 
de 21. Estados que participaban en la negociación. Otro grupo de Estados, sostenf́a una posición alternativa. Aún si el Tratado era firmado y ratificado por todos los Estados que participaban en la Comisión, este cuerpo legal no podía entrar en vigencia en tanto y cuanto no se completaran algunos requisitos básicos: la firma y ratificación del Tratado y sus dos Protocolos Adicionales por todos

Cuadro No I

ESTADOS QUE PARTICIPARON EN LA NEGOCIACION DEL TRATADO

\begin{tabular}{|c|c|c|}
\hline & ARGENTINA & 1964 \\
\hline & BOLTVIA ${ }^{x}$ & 1964 \\
\hline & BRASILI & 1964 \\
\hline & GHII,E $E^{1}$ & 1964 \\
\hline & COLOMBIA & 1964 \\
\hline & COSTA RICA & 1964 \\
\hline & ECUADOR ${ }^{1}$ & 1964 \\
\hline & GUATEMALA* & 1965 \\
\hline & HAITI ${ }^{2}$ & 1964 \\
\hline & HONDURAS & 1964 \\
\hline & JAMAICA & 1966 \\
\hline & $\mathrm{MEXICO}^{1}$ & 1964 \\
\hline & NICARAGUA & 1984 \\
\hline & PANAMA $^{ \pm}$ & 1964 \\
\hline & PARAGUAY & 1964 \\
\hline & PERU' & 1964 \\
\hline & REPUBLICA DOMINIGANA & 1964 \\
\hline$\ldots$ & TRINIDAD Y TOBAGO & 1966 \\
\hline & URUGUAY & 1964 \\
\hline & VENEZUELA" & 1966 \\
\hline
\end{tabular}

${ }^{\text {ICOPREDAL }}$ Y REUPRAL.

"REUPRAL.

Fuente: Informes y Declaraciones de Secretarios Generales $x$, In, in y IV Perfodos de Sesiones 1969-1975. Documentos Oficiales de la Conferencia General opANAI, Anexo $I_{,} p$. 75. 
los Estados que debian firmarlos y ratificarlos; y la conciusión de los acuerdos bilaterales o multilaterales con el Organismo Internacional de Energía Atómica destinados a asegurar el efectivo control de las actividades nucleares en todos los paises de la región.

Esta diferencia de criterios fue salvada en COPREDAL a través del diseño de una fórmula de conciliación que combinó ambas posturas estableciendo un sistema sui generis de entrada en vigencia que fue incorporado al Tratado en el Artículo 289.

Aun cuando esta solución de compromiso logró consolidar el régimen de desnuclearización que se pretendía establecer, no es menos cierto que con ello se permitió que algunos países de la región no se comprometieran plenamente con el Tratado y de hecho no se diera cumplimiento al requisito fundamental de la adhesión de la mayoría de los páses o al menos de aquellos militarmente más relevantes.

Es así como luego de transcurridos casi 20 años desde su firma en 1967, la situación de la NWFZ latinoamericana aún no se encuentra del todo Iograda. Cuba, país militarmente relevante, permanece al margen del Tratado, Argentina firmó y no lo ha ratificado, y Brasil y Chile lo suscribieron y ratificaron sin dispensa de los re- quisitos del artículo 28.

\section{Persistencia de los acuerdos preexistemtes entre las partes}

Como hemos señalado anteriormente, la iniciativa de establecer una zona desnuclearizada en América Latina a través de un pacto regional que contara con garantia de respeto por parte de las potencias extrarregionales intentaba ser un paso en el camino de desvincular la seguridad regional de problables enfrentamientos surgidos por motivaciones externas. No obstante ello, existía en la mayoría de los páses de Latinoamérica un rígido alineamiento con los Estados Unidos que se había expresado en el Tratado Interamericano de Asistencia Recíproca (TLAR) y cuya vigencia resultaba muy difícil de poner en duda. Numerosas críticas se han planteado en torno a la eficacia de este Tratado para garantizar la seguridad regional, argumentos que se fortalecieron además, a raíz del conflicto de las Malvinas de 1982. Pero hasta la fecha las proposiciones planteadas para su eventual reemplazo no han logrado imponerse y el TIAR persiste todavía como el mecanismo fundamental de seguridad regional.

En el terreno de la proliferación de las armas nucleares, Estados Unidos consideraba conveniente para sus intereses globales de seguguridad, evitar la diseminación de armas atómicas hacia nuevos Estados, ámbito en el que también podía encontrarse comunidad de

IIbid, p. 10.

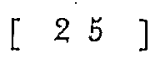


intereses con la URss ${ }^{10}$. De manera que là constitución de una NwFZ latinoamericana podia compatibilizar adecuadamente la persistencia de una alianza estratégica con los países de la región a través de la cual se pudieran controlar posibles amenazas externas en América Latina, con un esquema de desnuclearización total y definitiva que pudiera consagrarle una supremacía incontestable en el terreno de las armas nucleares en el continente americano.

\section{Sistema de Control confiable}

En cuanto a la necesidad de constituir un sistema de control confiable, el consenso entre los países se obtuvo ya en la segunda reunión de COPREDAL (23 de agosto al 2 de septiembre de 1965), lo que constituye un claro contraste con las dificultades que suelen presentarse en todas las negociaciones de acuerdos sobre desarme en que este punto suele ser el más controvertido.

En la primera reunión de la Conferencia General de las partes, el entonces Secretario General de las Naciones Unidas, U Than manifestaba:

"El Tratado de Tlatelolco es único, porque se refiere a una importante zona habitada de la tierra. También es único porque el organismo que se crea en este período de sesiones tendrá la ventaja de poseer un sistema permanente y eficaz de control con una serie de caracteristicas novedosas. Además de aplicar el sistema de medidas de protección del Organismo Internacional de Energía Atómica, el régimen establecido por el Tratado contiene disposiciones sobre informes e investigaciones especiales $y$, en caso de sospecha, inspecciones especiales. Este Tratado incluye una serie de aspectos del sistema conocido con el nombre de "verificación por desafío", que es uno de los conceptos nuevos que más esperanzas suscitan en la complicada cuestión de la verificación y control"11.

Es precisamente en este aspecto, en el que existió consenso entre las partes, donde nuevamente se requeriría de la concertación politica regional para superar la reticencia de Argentina para incorporarse plenamente al Tratado de Tlatelolco.

Argentina es el pafs que posee el mayor desarrollo nuclear en la región latinoamericana. La Comisión Nacional de Energía Atómica fundada.en 1950 ha impulsado permanentemente un programa de

${ }^{10}$ De hecho ambas superpotencias fueron propulsoras del Tratado de No Prolifexación de lạs Armas Nucleares y han continuado impulsándolo decididamente. IAEA, International Safeguards and the Non Proliferation Treaty. IAEA/PI/A 11. E.85-00942; abril 1985, p. 10.

11. Disscurso propunciads por el Secretario General de Naciones Unidas U Than en el Primer Perfodo de Sesiones de la Conferencia General. Documentos Ofi- 
perfeccionamiento humano y de adquisición de infraestructura física, basado en un proyecto destinado a dotar a la Argentina de un ciclo nuclear autónomo e independiente. La persistencia en los propósitos señalados y su materialización, hacen que la incorporación de este país se considere un elemento esencial para la consolidación de la NWTE.

En los últimos años la argumentación de Argentina se ha centrado en torno al sistema de salvaguardias que debe aplicar el Organismo Internacional de Energía Atómica. En efecto, entre la OIEA y la República Argentina se plantean serias diferencias en relación al tipo de salvaguandias que deben aplicarse en cumplimiento de las disposiciones del Tratado de Tlatelolco y Jas que son aplicables en función del Tratado de No Proliferación de las Armas Nucleares (TNP).

Se ha avanzado en las interpretaciones del TNP en el sentido de establecer salvaguardias internacionales a todo el ciclo nuclear, incluso a aquellas instalaciones que hayan sido autónomamente desarrolladas con el fin de controlar plenamente las posibles fuentes de desviación de material o instalaciones que puedan producirse. Al respecto el OIEA ha interpretado el Tratado de Tlatelolco en el mismo sentido y se han hecho dec.araciones por sus principales directivos en el sentido que "El Sistema de Salvaguardias parece ser igualmente aplicable al cumplimiento del Artículo 13 del Tratado de Tlatelolco, que asegura la aplicación de criterios internacionales y uniformes a situaciones que desde el punto de vista de las salvaguardias son prácticamente idénticos"12.

Para Argentina el problema se plantea en torno a la transferencia de tecnologia y los controles que los paises poseedores de tecnologia imponen a los países compradores: ... "desde un principio, el Organismo Internacional de Energía Atómica (OrEA), sacando provecho de que numerosas naciones latinoamericanas ya eran signatarias de ambos instrumentos (TNP y Tlatelọ ${ }^{1} \mathrm{co}$ ) impuso, presionado por las grandes potencias nucleares, un modelo de acuerdo de salvaguardias en las negociaciones con cada una de ellas en cumplimiento al Artículo 13 del Tratado, que respondia a los compromisos con-

ciales de la Conferencia General. Primer Período de Sesiones (Primera Parte, Suplemento No 1, OPANAL s/I), p. 42.

2Discurso del Representante del Organismo Internacional de Energfa Atomica Dr. Reinhard Rainer ante el Segundo Perfodo de Sesiones de la Conferencia General del opanal, Documentos Oficiales de la Conferencia General. Segundo Perfodo Ordinario de Sesiones, Suplemento NP 1 (s/5) p. 30. "I would like to adress to the safeguards which four of the five nuclear weapon states have spontaneously invited the IAEA to carry out in respect of facilities or fissile material in their peaceful nuclear energy sectors. The USA, The United Kingdom, France, and several months ago the Soviet Union have concluded safeguards agreements of this kind with the IAEA". "Oụr intention has been to counter 
trafdos en virtud del xNe, y no a uno especialmente elaborado para ajustarse al espíitu y a la letra del Tratado de Tlatelolco"18.

Según el Artículo 13, los países miembros del Tratado de Tlatelolco deben negociar y concluir Acuerdos de Salyaguardias con el Organismo Internacional de Energía Atómica, obligación que no está condicionada o subordinada a la existencia o planeación de una actividad nuclear por parte del Estado involucrado. "Todo Estado Parte en el Tratado de Tlatelolco, aunque no desarrolle actividades nucleares ni planifique realizarlas, tiene el deber de concluir estos Acuerdos como lo señaló expresamente la Resolución 51 (III) de la Conferencia General, adoptada el 23 de agosto de 1973"14.

Corresponde al OPANAL controlar el cumplimiento de la obligación prevista en el artículo 13, y a este organismo se le ha atribuido también la facultad de negociar, en representación de los pafses miembros que lo deseen, los acuerdos que deben suscribir con el OIEA.

Si se considera que existe real consenso entre los países latinoamericanos sobre la necesidad de preservar la zona desnuclearizada, y que este objetivo implica necesariamente la existencia de mecanismos de control y verificación que aseguren el efectivo cumplimiento de las obligaciones previstas en el Tratado; si tenemos en cuenta que el Tratado de Tlatelolco señala expresamente la obligación de concluir acuerdos de salvaguardias con el OrEA, sea cual fuere el nivel de desarrollo nuclear de cada parte, y que Argentina no acepta la adaptación automática de los acuerdos de salvaguardias que el OIEA ha desarrollado para los países firmantes del TNP, correspondería al OPANAL intentar un proceso de negociación que pudiera acercar ambas posiciones.

Es evidente que cualquier sistema de salvaguardias que se intente establecer debe llenar el requisito fundamental de servir de garantía del efectivo cumplimiento de la obligación fundamental de mantener toda actividad nuclear que se desarrolle en América Intina enmarcada dentro del terreno de los usos pacíficos, proporcionando a todos los Estados de la región la seguridad permanente y

some of the criticism which says that the nuclear weapon States have benefitted by being spared the expense and intrusion inspection entails". Hans Blix, Safeguards and the NPT, en IAEA Bulletin, Vol. 27, NO 2, Summer 1985, p. 7. Vex también IAEA, International Safeguards...", op. cit. p. 13.

1"Declaraciones del Observador de la República Argentina, señor José Maria V. Otegui, en el Séptimo Período Ordinario de Sesiones de la Conferencia General, Documentos Oficiales de la Conferencia General, Suplemento No 1 (s/25), p. 46. Ver también Foro Interdisciplinario de Política Nuclear, Reflexiones y Recomendaciones de la Comisión de Política Nuclear Internacional, 21-29 de mayo de 1984, publicado en Revista Argentina de Estudios Estratégicos, año $1, N^{\circ} 1$, julio-agosto-septiembre I984, p. 160.

"Héctor Gros Espiell, El tratado de Tlatelolco, diez años de aplicación, 19671977, OPANAL, México, 1978, p. 86. 
completa de que el compromiso con la desnuclearización persiste, situación que se torna cada vez más complicada en la medida en que los países miembros van desarrollando planes ambiciosos de utilización de la energía nuclear.

Si se alienta un proceso de discusión en el ámbito del OPANAL sobre el alcance de las salvaguardias que deben exigirse a los Estados latinoamericanos, en el que puedan participar especialistas de la región que evalúen técnicamente las reservas de Argentina en relación al sistema de control del oIEA, podrán eventualmerite negociarse las adaptaciones que resulten necesarias para establecer un sistema de salvaguardias adecuado a las exigencias de la Nwrz latinoamericana.

Una negociación de esta naturaleza en el seno del opANAL, aparte de satisfacer el objetivo inmediato de allanar el camino para que Argentina materialice definitivamente su proceso de ratificación del Tratado de Tlatelolco, podría contribuir a vitalizar el Organismo regional dotándolo de capacidad para servir de foro de concertación política en el terreno de la cooperación latinoamericana en el campo de la energía nuclear.

\section{Los usos pacificos de la energía muclear deben estar permitidos}

El requisito de permitir los usos pacíficos de la energía nuclear se encuentra expresamente consagrado en el artículo 17 del Tratado de Tlatelolco. En la práctica los países latinoamericanos han diseñado programas de desarrollo nuclear fundados en consideraciones energéticas y paralelamente en percepciones asociadas al incremento del poder nacional. Tradicionalmente se ha mirado al progreso en el campo de la energía nuclear como una demostración efectiva de un nivel de excelencia en el plano cientifico, tecnológico e industrial.

En el caso argentino, el programa nuclear se inició con mucha antelación al del resto de los países latinoamericanos. La 'Comisión Nacional de Energía Atómica se fundó en 1950 bajo Ios auspicios del Gobierno del General Domingo Perón. Desalentado por las críticas que habia suscitado el proyecto del científico austríaco Ronald Richter, quien lo había hecho creer infundadamente, que contaba con la capacidad suficiente como para construir en forma autónoma en Argentina una bomba de fusión, resolvió entonces establecer un organismo nacional que incorporarfa cientfficos argentinos que elaborarfan un programa de desarrollo autosuficiente para el país ${ }^{15}$.

Las expectativas iniciales del Presidente Perón, que ligaron el desarrollo nuclear a la perspectiva de dotar a Argentina de capacidad

${ }^{20} J$ José Federico Westerkamp, "La energia nuclear: Relevancia y Perspectivas para América Latina y Estados Unidos", en: Cuadernos Semestrales, No 15, Me- 
atómica bélica sembraron la desconfianza regional sobre los verdaderos objetivos del proyecto. Durante los últimos 35 años Argentina ha continuado desarrollando ininterrumpidamente un programa nuclear ambicioso, que lo ha conducido al liderazgo indiscutido en la región. En la actualidad, el país cuenta con reactores de potencia, reactores de investigación, ha ensayado un procedimiento de enriquecimiento de uranio, y se ha empleado en el desarrollo de un proceso autónomo de fabricación de agua pesada y reprocesamiento. A estos avances en el plano científico y técnico, sumamos el hecho de que la GNEA se ha mantenido como una organización estable con financiamiento prioritario entre los organismos nacionales, desafiando las tumultuosas variaciones politicas del sistema de gobierno ${ }^{16}$.

En el caso de Brasil, el énfasis en el programa nuclear se produjo a partir de la crisis del petróleo, que provocó un fuerte impacto en las expectativas de crecimiento económico del país, en la medida en que Brasil aparecía como altamente vulnerable por su extrema dependencia externa para el abastecimiento de energéticos. Como el factor decisivo en este caso era el de la vulnerabilidad extema, el proyecto brasileño también buscó con especial interés la autonomfa de su programa nuclear ${ }^{17}$.

La decisión brasileña fue particularmente difícil, porque precisamente en este período se produjo una readecuación muy significativa del sistema de proveedores nucleares internacionales, motivado fundamentalmente por la utilización por parte de la India de tecnología y equipos de procedencia canadiense para la fabricación del artefacto nuclear que hiciera explotar en 1974. A pesar de que la India expresó reiterativamente que su explosión nuclear había sido pacífica, los países proveedores comprobaron como un hecho indesmentible que sus transferencias nucleares podfan servir de base a la temida proliferación nuclear. A ese período corresponden los in-

xico, ler. Semestre 1984. Revista Argentina de Estudios Estratégicos, año 1, No 1, julio-agosto-septiembre, 1984. Daniel Poneman, "Nuclear Proliferation, Prospects for Argentina, en ORBIS, Winter 1984, p. 853. El retorno a la democracia también ha afectado a la CNEA, que es actualmente dirigida por un funcionario civil. Tradicionalmente el director habla sido un marino de la más alta jerarquia que ocupaba el cargo por largos perfodos de tiempo, en agudo contraste con la inestabilidad política del pals. Asimismo las dificultades financieras también han alcanzado a los' programas nucleares de Argentina y Brasil. Las instalaciones en construcción .se han visto seriamente retrasadas, los empleados y obreros han debido llegar a la huelga para exigir atención a sus problemas laborales, $y$ en el caso argentino se ha bablado de una irreparable fuga de cerebros. Latin American Weekly Report, wR-86-16, 25 de abril de 1986, p. 3 y wR86-08, 17 de enero de 1985, p. 5.

Ifosé Federico Westerkamp, "Energia Nuclear...", op. cit. p. 148. ITbid. 
tentos de aglutinar en un club secreto a los proveedores y fijar en conjunto normas de transferencia que evitaran la competencia desleal entre las empresas abastecedoras y permitieran un eficaz control de la proliferación mediante un rígido sistema de salvaguardias aplicado por la OIEA ${ }^{18}$.

La necesidad de vender tecnología y equipos pudo más que cualquier concertación y Brasil firmó con Alemania Federal un contrato de enormes proporciones abarcando desde la fabricación de reactores nucleares, a instalaciones de enriquecimiento y aún de reprocesamiento. Este acuerdo contó con la decidida oposición del Gobierno norteamericano, que en ese perfodo restringió decisivamente sus condiciones de venta de tecnología y equipos, colocando a su industria nuclear en una seria desventaja competitiva que debịó ser corregida posteriormente. De este modo, la industria nuclear norteamericana no logró aprovechar los buenos momentos del shock petrolero, que una vez superados por el avance de nuevas tecnologias, las medidas de conservación energética y el encarecimiento de las plantas nucleares por mayores requerimientos de seguridad, entro en una etapa de estancamiento. Desde luego estos elementos espectficos ligados a la industria energética han estado enmarcados pór los efectos recesivos de la economía internacional en su conjuntón

En el caso de Chile, que también venía desarrollando un interesante proyecto nuclear, y que había realizado estudios de eñplazamiento para sus futuras centrales de potencia, alentado por el informe de factibilidad de la orea, que había señalado a Chille comó un país en el que resultaba posible y deceable instalar este tipo de centrales energéticas, el gobierno acordó dilatar esa opción teniendo como base un estudio realizado por la Comisión Nacional de' Energía, que demostraba la mayor eficiencia económica de continuar con el desarrollo energético a través de centrales hidroeléctricas ${ }^{20}$.

Cuba, es un caso excepcional, debido a la estrecha dependencia de su programa nuclear de las transferencias soviéticas, particularidad que se repite además en el ámbito de la seguridad, como también en su posic'ón en torno al Tratado de Tlatelolco ${ }^{21}$.

México, que por tamaño y desarrollo habría tenido posibilidades

\footnotetext{
${ }^{28}$ Arturo Alessandri, Pilar Armanet y Francisco Orrego, "El TNP y las nuevas alternativas de Política Nuclear", en: Francisco Orrego y Pilar Armanet (eds.) Politica Nuclear, Colección Estudios Internacionales, Editorial Universitaria, Santiago, 1979, p. 27 y ss.

${ }^{19}$ Consejo Atlántico de los Estados Unidos: La Energia Nuclear y la Proliferación de Armas Nucleares, Noema Editores, México, 1979, p. 47.

sonforme de la Comisión Nacional de Energía al Gobierno de Chile, marzo 1980. (Circulación restringida).

-José Westerkamp, "La Energía Nuclear, Relevancia y Perspectivas...", op. cit, p. 158 .
} 
de emprender un programa nuclear ambicioso, ha preferido privilegiar otros aspectos de su programa energético, fundado tanto en sus disponibilidades de petróleo como en su decis ón política de apoyar la desnuclearización regional, elementos que han contribuido a mantener a México en el plano atómico por debajo de sus potencialidades en otros campos ${ }^{22}$.

Otras experiencias interesantes en América Latina están ligadas a la cooperación. En este sentido, particular interés se asigna a la colaboración prestada por Argentina al desarrollo nuclear de Perú y a los acuerdos con Uruguay, Brasil y Chile, que muestran un camino de acercamiento hacia iniciativas regionales que permitan aprovechar economías de escala para instalaciones y equipos diseñados generalmente para requerimientos mucho mayores que los que presentan los países latinoamericanos individualmente considerados. Paralelamente, ello implica una posibilidad para los países que han realizado esfuerzos por desarrollar programas nucleares autónomos, de recuperar en parte el costo de investigación y realización de esos proyectos ${ }^{23}$.

En resumen, en la región latinoamericana el desarrollo nuclear ha obedecido a requerimientos energéticos y de prestigio internacional, variables que han estado siempre presentes, aun cusndo han pesado en forma diferente en cada uno de los casos. En definitiva puede afirmarse que la existencia del compromiso de mantener la región desnuclearizada, con todas las imperfecciones que el régimen establecido pueda haber tenido desde sus inicios, contribuyó a un cierto mínimo común denominador en el sentido de clarificar el escenario regional, ya que periódicamente los países fueron haciendo presente que persistían en su decisión de respetar los principios del Trataro de Tlatelolco ${ }^{24}$. Este hecho es particularmente significativo si se tiene en cuenta que las declaraciones han correspondido a paises que como Argentina, Brasil y Chile no han configurado jurídicamente su total adhesión al Tratado y que son precisamente los que tienen programas nucleares más desarrollados ${ }^{25}$.

Aun cuando, obviamente esta no ha sido la única causal a la que puede atribuirse la ausencia de una proliferación horizontal en América Latina, ello ha concedido a la región una configuración especial que puede contribuir a facilitar iniciativas de seguridad

2Ibid, .pp 151-52.

${ }^{23}$ John Redick, "The Tlatelolco Regime and Non-Proliferation in Latin America", en: International Organization, vol. 35, No 1, winter 1981, p. 127.

${ }^{24}$ José Martinez Cobo, América Latina como Zona Desnuclearizada, presentatado al "Seminario sobre Seguridad y Paz en América Latina". Instituto de Estudios Internacionales, Universidad de Chile, Viña del Mar, $19-22$ de noviembre de. 1984.

mpilar Armanet, "Energfa Nuclear y Seguridad Regional. Proposiciones para 
más ambiciosas, como podría ser la de constituirse en una etapa pionera en la construcción de una zona de paz en América Latina.

\section{Delimitación de un espacio geográfico de aplicación}

El Tratado de Tlatelolco es explícito en la definición de su ámbito geográfico de aplicación, según el cual las partes contratantes del Tratado, esto es, aquéllas para las cuales el Tratado está en vigor (artículo 2) se comprometen a impedir y prohibir en sus respectivos territorios "el ensayo, uso, fabricación, producción o adquisición, por cualquier medio, de toda arma nuclear, por si misma, directa 0 indirectamente, por mandato de tercero o en cualquier otra forma" y "el recibo, almacenamiento, instalación o emplazamiento o cualquier forma de posesión de toda arma nuclear, directa o indirectamente, por sí mismas, por mandato de terceros o de cualquier otro modo". Además "deberán abstenerse de realizar, fomentar o autorizar, directa o indirectamente, el ensayo, el uso, la fabricación, la producción, la posesión o el dominio de toda arma nuclear o de participar en ello de cualquier manera"26. Todas estas actividades, están expresamente proscritas en el territorio de los Estados miembros, que "incluye el mar territorial, el espacio aéreo y cualquier otro ámbito sobre el cual el Estado ejerza soberanía según su propia legislación" (artículo 3). Estas disposiciones implican en consecuencia, que el ámbito geográfico del Tratado se configura por la suma de los territorios de los Estados que han adherido al Tratado de Tlatelolco (artículo 4, párrafo 1). No obstante, una vez reunidos todos los requisitos del artículo 28 , esto es, que todos los Estados a los cuales el Tratado y los dos Protocolos adicionales los hayan suscrito y ratificado, el espacio territorial de aplicación será más amplio que la simple suma de los territorios de todos los Estados, ámbito que se delimita expresamente en el artículo 4 párrafo $2^{27}$.

Los Estados nucleares y en generaI los patses poseedores de poderosas flotas han expresado su preocupación por las posibles limitaciones que pueden imponerse al libre tránsito en determinados espacios marítimos. Esta percepción se ha agudizado ante la tendencia a ampliar los derechos jurisdiccionales de los Estados costeros a zonas que anteriormente se encontraban sujetas al régimen de alta mar, y principalmente por el hecho de que algunos Estados han interpretado la zona económica exclusiva consagrada por la Convención de Jamaica de 1982, como una zona de mar territorial en la

América Latina", en Gustavo Lagos (ed.), No al Apocalipsis Nuclear, Achip, Editorial Salesiana, Santiago, 1984, pp. 165-67.

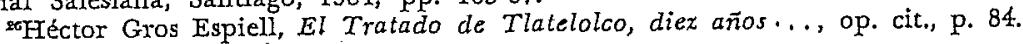

Thid, p. 85. 
que los países ribereños tendrían derechos similares a los que se reconoce para el mar territorial propiamente tal, que se extiende solamente a 12 millas marinas. Si a estas diferencias de interpretación que en algunos casos han sido recogidas por la legislación interna de ciertos Estados, agregamos la preocupación causada por la decisión de Nueva Zelandia de prohibir el ingreso a sus aguas territoriales y a sus puertos de buques portando armas nucleares y aún de aquellos que no portándolas son propulsados por energía nuclear, las dificultades pueden ser todavía mayores ${ }^{28}$.

En el caso de Tlatelolco, el Tratado no prohíbe expresamente el tránsito de armas nucleares. Aunque no es imposible una interpretación del Tratado que conduzca a un resultado prohibitivo. De hecho, ante las protestas argentinas por la incursión de buques $y$ submarinos portando armas atómicas, y propulsados por energía nuclear, el Secretario General del opanal expresó su opinión en el sentido de que un buque de guerra propulsado por energfa nuclear que participaba en un conflico bélico, difícilmente podría considerarse que estaba utilizando la energía nuclear con fines pacfficos, $v$ que en consecuencia con ello se estaban violando los objetivos y fines del Tratado que constitúa la zona desnuclearizada ${ }^{29}$.

La Comisión Preparatoria que redactó el texto del Tratado incluye una interpretación en materia de tránsito, según la cual "si el transportador fuese de las partes contratantes, el transporte queda cubierto por las prohibiciones del artículo l" y "si el transportador fuese un Estado que no sea parte en el Tratado, el transporte se identifica con el tránsito respecto al cual, no existiendo en el Tratado ninguna disposición, debe entenderse que se aplicarán los principios y normas del Derecho Internacional en la materia, según los cuales corresponde al Estado territorial, en el libre ejercicio de su soberanfa, otorgar o negar dicho tránsito"30. Esta interpretación. ha sido reconocida como válida por Gran Bretaña, Francia y tos Estados Unidos, y así lo manifestaron en sus declaraciones interpretativas al momento de suscribir y ratificar los Protocolos Adicionales. La uRss ha sostenido la opinión contraria: "la autorización de tránsito de armas nucleares en cualquier forma serfa contraria a los fines del Tratado, según el cual. como se señala especialmente en su preámbulo, la América Latina debe ser completamente libre de armas nucleares, y sería incompatible con el estatuto de desnuclearización de los Estados Partes en el Tratado y con sus obligaciones determinadas por el artículo I del Tratado"81.

${ }^{23}$ Rqmesh Thakur, "A Nuclear Weapon Free Zone...", op. cit., p. 229.

20Informe del Secretario General, OPANAL, CG/234, 15 de abril de 1983, p. 8.

roóéctor Gros Espiell, El Tratado de Tlatelolco; diez años..., op. cit., p. 85, y p. 112.

arHéctor Gros Espiell, "Reservas y Protocolos Adicionales al Tratado de Tla- 
En la etapa actual del desarrollo de las marinas de guerra resulta difícil de aceptar para una superpotencia como Estados Unidos, interpretaciones excesivamente restrictivas del Tratado de Tlatelolco que puedan entorpecer el tránsito de gran parte de sus buques y submarinos propulsados por energía nuclear y portadores de armas atómicas, a ello se suma el hecho de que razones de estrategia militar son generalmente esgrimidas para negar información detallada sobre el tipo de armamento que Ileva a bordo una flota de guerra. Para los paises latinoamericanos las disposiciones sobre navegación reconocidas por el derecho internacional resultan plenamente aplicables en este caso, permitiéndose en consecuencia, la libre navegación en las 200 millas de zona económica exclusiva y el paso inocente en el mar territorial ${ }^{32}$. Dentro de este marco general cada país podrá, a través de sus leyes internas, reglamentar el paso inocente en sus aguas territoriales y en el acceso a sus puertos, para lo cual deberá tomar en cuenta sus intereses naciona'es de seguridad resolviendo en la mejor forma en que éstos puedan garantizarse.

\section{Apoyo de las potencias nucleares}

Desde que se iniciara en la década de los 60 el esfuerzo pór constituir en América Latina una NWFZ existió acuerdo entre los países latinoamericanos respecto a la necesidad de respaldo por parte de las potencias nucleares y la comunidad internacional.

Estas ideas motivaron el esfuerzo diplomático y juridico de sus inspiradores y se materializaron en un conjunto de resoluciones de Naciones Unidas que reconocieron la val:dez y eficacia de las zonas desnuclearizadas como elementos contribuyentes a consolidar la paz y promover el desarme, y más concretamente, en el propio Tratédo de Tlatelolco en la inclusión de dos Protocolos adicionales destinados a las potencias extra-regionales ${ }^{33}$.

La decisión de anexar al Tratado de Tlatelolco dos Protocolos, para exigir el compromiso de las potenc: as nucleares a respetar la zona desnuclearizada, aparece además, como una expresión de un adecuado equilibrio entre las obligaciones de pafses nucieares y no nucleares. El Protocolo ir exige de las potencias nucleares extracontinentales la obligación de no usar ni amenazar con el uso de armas atómicas a los países de América Latina y ha sido ya suscrito y ratificado por las cinco potencias nucleares reconocidas. El Pro-

telolco", en:Revista Argentina de Relaciones Internacionales, No 13, enero/abrii 1979 , p. 87.

agulio César Lupinacci, "La Naturaleza Jurfdica de la Zona Económica Exclusiva", en Francisco Orrego (ed.), La Zona Económica Exclusiva, una Perspectiva Latinoamericana", Colección Estudios Internacionales, Instituto de Estudios Internacionales, Universidad de Chile, Santiago 1982.

@esolución 2028 de la Asamblea General de las Naciones Unidas. 
tocolo $\mathrm{I}$, exige que las potencias extrarregionales que poseen de jure - de facto territorios en América Latina deben comprometerie a mantener alejadas de esos territorios las armas nucleares. Todas las potencias nucleares que se encuentran en estas condiciones, con excepción de Francia, han suscrito y ratificado el acuerdo ${ }^{34}$.

Cuadro No 2

PROCESO DE FIRMA Y RATIFICACION DE LOS PROTOCOLOS I Y II DEL TRATADO DE TLATELOLCO

\section{Protocolo Adicional I}

Firma

Estados Unidos, 26 de marzo 1977

Francia, 2 de marzo 1979

Gran Bretafía, 20 de diciembre 1967

Pafses Bajos, I5 de marzo 1968
Ratificación

29 de noviembre 1981

II de diciembre 1969

26 de julio 1971

PROTOCOLO ADIGIONAL II

China, 21 de agosto 1973

Estados Unidos, 19 de abril 1968

Francia, 18 de julio 1973

Gran Bretaña, 20 de diciembre 1967

Unión Soviética, 18 de mayo 1978
12 de junio 1974

12 de mayo 1971

22 de marzo 1974

11 de diciembre 1969

12 de diciembre 1978

\section{Conclustones}

Luego de 20 años de vigencia siguen existiendo fundadas dudas respecto a la real eficacia del Tratado de Tlatelolco para establecer una zona desnuclearizada. Las principales amenazas de que este proyecto se debilite y probablemente nunca se complete, provienen de la falta de compromiso de ciertos países que como Argentina, Brasil, Chile y Cuba aún no han dado todos los pasos para comprometerse definitivamente con la desnuclearización latinoamericana.

s1Pilar Armanet, "Energla Nuclear y Seguridad Regional...", op. cit. 
Brasil y Argentina señalaron inicialmente que sólo serían parte integral del Tratado cuando todos los países de la región y las potencias extrarregionales a las cuales el Tratado les fuera aplicable, lo hubieran efectivamente suscrito y ratificado. A ese primer requisito se han comenzado a agregar las dificultades ya analizadas, que se refieren al problema de la transferencia de materiales y equipos que requieren estos países para el pleno desarrollo de sus programas nucleares. Los controles a la transferencia que establecen los países proveedores han sido considerados como mecanismos de presión y restricción indebida a la transferencia de tecnología nuclear y los principales interesados en impulsar a Argentina y Brasil a suscribir el Tratado y firmar acuerdos de salvaguardjas han sido precisamente los países proveedores. Argentina, principalmente, ha sostenido que sus dificultades para obtener tecnologia han impulsado al pars hacia un desarrollo autónomo y que en consecuencia difícilmente pueden exigirsele salvaguardias internacionales aplicables por el Organismo Internacional de Energía Atómica. Asimismo se han señalado críticas al oIEA relativas al secreto industrial que sería seriamente amenazado por el sistema de salvaguardias, dificultando aún más las posibilidades de que los controles externos puedan apicarse $^{35}$.

El problema a nuestro juicio reside fundamentalmente en una confusión en torno al verdadero destinatario de la desnuclearización latinoamericana, que ha sido y sigue siendo la propia seguridad regional. En la medida en que algunos países condicionen su adhesión a sus dificultades en el plano de la tecnología o el secreto industrial y aún de la negociación con países extrarregionales, la desnuclearización de la región comienza a convertirse en un proyecto irrealizable.

En el caso de Guba, su compromiso se encuentra supetitado a sus relaciones de confrontación con los Estados Unidos, y en consecuencia resulta difícil imaginar un mejoramiento de esa contradicción. La perspectiva de que Estados Unidos abandone sus bases militares en la Isla y se emplee en una efectiva desnuclearización son objetiros muy lejanos que escapan a los intereses de seguridad meramente regionales.

En el caso chileno, inicialmente se asoció la adhesión al Tratado al requerimiento de que simultáneamente se comprometieran plenamente Brasil y Argentina. Posteriormente, se condicionó la posición chilena a la suscripción y ratificación del Protocolo II por parte de la URss, hecho que sucedió, sin que Chile modificara su actitud reticente. A la fecha, pareciera no existir elemento a'guno que justi-

"Daniel Poneman, "Nuclear Proliferation Prospects for Argentina", ORBIS, vol. 27, No 4, winter 1984 . 
fique la posición chilena, ya que su principal seguridad, aún en un escenario de confrontación, provendría del convencimiento de que en América Latina no existen armas nucleares, porque el país está muy lejos de poder desarrollarlas en forma autónoma ${ }^{36}$.

La crisis de los sistemas de seguridad hemisférica dramáticamente expresada en el conflicto del Atlántico Sur reabrió el debate è América Latina respecto a la necesidad de revitalizar la zona desnuclearizada como un germen existente, aun cuando débil de una zona de seguridad regional compartida. El opANAL fue teatro de controvertidas sesiones en las que los paises miembros apoyaron a Argentina en su protesta contra Gran Bretaña, fundada en la acusación de que en el conflicto de las Malvinas, Inglaterra habría traido armas nucleares a la zona en abierta contradicción a sus compromisos contraídos con la región y concretamente sus obligaciones en virtud de los Protocolos I y II del Tratado de Tlatelolco. Los países miembros del Tratado en una Resolución que ha sido considerada algo neutra y poc odefinida, ya que solamente "tomó nota" de la declaración argentina y "expresó su preocupación" por la utilización de submarinos atómicos, implicó un paso adelante en el proceso de concertación política del opANAL como un foro adecuado para discutir y generar una postura latinoamericana en materias de seguridad.

Creemos que en la medida en que los países latinoamericanos revitalicen dentro del opaNal y en los demás foros regionales los objetivos que se tuvieron presente al momento de crear la zona desnuclearizada y estén dispuestos a perfeccionarla y apoyarla políticamente, el proyecto que se ideó en los 60 puede llegar a una efectiva aplicación. Este escenario que es el más optimista sólo puede inspirarse en un sistema de relaciones futuras fundadas en la cooperáción regional y el respeto de las potencias extra-regionales, en la medida en que se comiencen a establecer las condiciones para ello, la zona desnuclearizada podrá ser considerada como un primer paso ya iniciado para el establecimiento de un esquema regional de seguridad compartida.

\footnotetext{
"Telegrama de 19 de agosto de 1975 enviado por el Canciller de Chile AImirante Patricio Carvajal al Secretario General del opanal, en: Informe del Secrelario General, mayo 1980-abril 1981. cG/203, 25 de mayo de 1981, p. 15.
} 